\title{
Do Parent-Adolescent Discrepancies in Family Functioning Increase the Risk of Hispanic Adolescent HIV Risk Behaviors?
}

\author{
DAVID CORDOVA* \\ SHI HUANG ${ }^{\dagger}$ \\ MEGHAN LALLY: \\ YANNINE ESTRADA ${ }^{\dagger}$ \\ GUILLERMO PRADO $\$$
}

In the family-based prevention science literature, family functioning, defined as positive parenting, parental involvement, family cohesion, family communication, parental monitoring of peers, and parent-adolescent communication, has been shown to ameliorate HIV risk behaviors in Hispanic youth. However, the majority of studies have relied solely on parent or adolescent reports and we know very little about parent-adolescent family functioning discrepancies. Therefore, the purpose of this study was to examine whether and to what extent parent-adolescent discrepancies in family functioning increased the risk of HIV risk behaviors, including substance use and sexual risk behaviors, and whether these associations vary as a function of acculturation and youth gender. A total of 746 Hispanic 8th grade youth and their primary caregivers were included in the study. Structural equation modeling findings indicate that parent-adolescent family functioning discrepancies are associated with an increased risk of Hispanic adolescent HIV risk behaviors, including lifetime and past 90-day alcohol and illicit drug use, and early sex initiation. In addition, study findings indicate that results vary by acculturation and youth gender. Findings are discussed in the context of existing family-based research and practice in preventing and reducing HIV risk behaviors among Hispanic youth and their families.

Keywords: Hispanic/Latino; Adolescents; Prevention; Family Functioning; Substance Use; HIV

Fam Proc 53:348-363, 2014

\section{INTRODUCTION}

$\mathrm{T}$ The human immunodeficiency virus (HIV) represents a major public health concern facing America's youth. Hispanic youth disproportionately engage in problem behaviors, including substance use and sexual risk behaviors that place them at increased risk

${ }^{*}$ School of Social Work, University of Michigan, Ann Arbor, MI.

${ }^{\dagger}$ Public Health Sciences, University of Miami Miller School of Medicine, Miami, FL.

${ }^{\ddagger}$ Psychology, Arizona State University, Tempe, AZ.

${ }^{\S}$ Public Health Sciences, University of Miami, Miami, FL.

Correspondence concerning this article should be addressed to David Cordova, School of Social Work, University of Michigan, 1080 S. University, Ann Arbor, MI (e-mail: cordovad@umich.edu) and Guillermo Prado, Department of Public Health Sciences, University of Miami Miller School of Medicine, 1120 N.W. 14th St., Suite 1010, Miami, FL 33136 (e-mail: gprado@med.miami.edu).

This study was supported by Grants \#R01 DA025192 (PI, Prado) and 3R01 DA025192S1 (PI, Prado). Preparation of this manuscript was supported in part by Grants\# 1L60MD006269-01 (PI, Cordova) and R25 DA026401 06 (PI, Valdez). Meghan Lally was supported by an NIMH training Grant\# T32 MH 018387 (PI, Laurie Chassin). 
of HIV acquisition and transmission (Centers for Disease Control \& Prevention, 2012a; Johnston, O’Malley, Bachman, \& Schulenberg, 2012). Findings from the Monitoring the Future study, for example, indicate that Hispanic 8th graders report the highest rates of lifetime alcohol and drug use, as compared with their African American and non-Hispanic White counterparts (Johnston et al., 2012). Furthermore, relative to African American (65.3\%) and non-Hispanic White youth (59.5\%), Hispanic youth (58.4\%) are least likely to report having used a condom during their last sexual encounter (Centers for Disease Control \& Prevention, 2012a). It should not be surprising then that Hispanics are disproportionately impacted by HIV. Despite accounting for $16 \%$ of the total U.S. population, in 2010, Hispanics accounted for 21\% of new HIV infections (Centers for Disease Control \& Prevention, 2012b), signaling a need for intervention.

The family system has been shown to be among the most influential at ameliorating HIV risk behaviors, including substance use and sexual risk behaviors, among Hispanic youth (Sandler, Schoenfelder, Wolchik, \& MacKinnon, 2011; Santisteban \& Mena, 2009; Szapocznik, Prado, Burlew, Williams, \& Santisteban, 2007). For example, higher levels of family functioning, defined as positive parenting, parental involvement, family cohesion, family communication, parental monitoring of peers and parent-adolescent communication, have been shown to prevent and reduce substance use and sexual risk behaviors in Hispanic youth (Córdova, Huang, Pantin, \& Prado, 2012; Prado, Córdova et al., 2012; Prado, Pantin et al. 2012). Yet, relatively little is known with respect to the impact of parent-adolescent family functioning discrepancies on HIV risk behaviors. That is, most research has relied solely on one perspective (e.g., parent report) of family functioning and has not examined parent reports in conjunction with adolescent reports and the difference in perspectives between the two reports. Therefore, the purpose of this study was to examine whether and to what extent parent-adolescent family functioning discrepancies increase the likelihood of Hispanic youth engaging in HIV risk behaviors, including substance use and risky sexual behaviors, and whether these associations vary as a function of acculturation and youth gender.

\section{HIV Risk Behaviors: Substance Use and Sex Risk Behaviors}

Hispanic youth disproportionately engage in HIV risk behaviors, including substance use and sexual risk behaviors. In addition to reporting higher rates of lifetime alcohol and drug use, parallel data indicate that Hispanic 8th grade youth are more likely to report current alcohol and drug use, as compared to both African American and non-Hispanic Whites (Johnston et al., 2012). In addition, findings from the Youth Risk Behavior Surveillance Survey suggest that, relative to non-Hispanic Whites (3.1\%), Hispanic (7.9\%) youth are more likely to report early sex initiation (Centers for Disease Control \& Prevention, 2012a). Taken together, this is disconcerting as both substance use and risky sex behaviors, including unprotected sex and early sex initiation, have been shown to increase risk for HIV acquisition and transmission (Prado et al. 2010).

\section{Etiology of HIV Risk Behaviors: Family Functioning}

A substantial number of studies have demonstrated the important role the family system has on the etiology of Hispanic youth HIV risk behaviors (Prado et al. 2010; Santisteban et al., 2003; Unger, Ritt-Olson, Wagner, Soto, \& Baezconde-Garbanati, 2009). Drawing from etiological research, family systems characterized by higher levels of family functioning, including family support, family communication, parent-adolescent communication, family bonding, parental monitoring of adolescents' peers, and family cohesion, have been shown to prevent and reduce HIV risk behaviors, including substance use and risky sex, among Hispanic youth (Córdova et al., 2011; Prado et al. 2010; Sale et al., 
2005). Intervention research has even identified family functioning as a pathway through which family-based preventive interventions impact adolescent HIV risk behaviors (Sandler et al., 2011). For example, studies show that the effects of Familias Unidas, a family-based preventive intervention, on substance use and sexual risk behaviors are partially mediated by improvements in family functioning (Prado et al., 2007; Prado, Córdova et al., 2012; Prado, Pantin et al. 2012).

\section{Empirical Research and Reports of Family Functioning}

While a number of empirical studies clearly indicate that family functioning is especially important in ameliorating Hispanic adolescent HIV risk behaviors, methodological approaches that include both parent and adolescent reports of family functioning are rare. This is surprising given that perceptions of the family system can vary by different family members. In fact, the most widely practiced methodological approach to assessing family functioning in empirical studies uses only a single report. Specifically, whereas some studies utilize parent reports to assess family functioning (e.g., Martinez \& Eddy, 2005; Prado et al., 2007), other studies utilize adolescent reports (e.g., Mouttapa, Weiss, \& Hermann, 2009; Sale et al., 2005). To date, only a limited number of studies have included both adolescent and parent reports of family functioning (Sullivan et al., 2007). There are at least two explanations for this; first, in many family-based preventive interventions, the parents are seen as the change agent and the majority of intervention activities are directed at the parent. Thus, it would seem logical to include parent reports on family functioning. Second, the agreement between informant reports of family functioning is often in the low to moderate range ( $r$ s ranging from .20 to .60; Achenbach, 2006), which poses a methodological challenge for researchers. This challenge has typically been addressed by electing to use the "most reliable" reporter given the construct of interest. While Tein, Roosa, and Michaels (1994) were among the first to question the practice of aggregating scores across reporters within the family, even they concluded that if the question of substantive interest concerned the youth outcomes, the adolescent's perceptions, even with biases, may count more than the parent's perceptions. This may be a particularly important assertion as adolescents tend to report less favorable views of family functioning, relative to parent reports (Ohannessian, Lerner, Lerner, \& von Eye, 1995; Schwartz, Mason, Pantin, \& Szapocznik, 2008; Steinberg, 1990; Stuart \& Jose, 2012).

\section{Parent-Adolescent Discrepancies of Family Functioning}

More recently, renewed interest in examining the impact of parent-adolescent family functioning discrepancies on adolescent problem behavior outcomes has emerged. This line of research has challenged the common practices of both excluding or aggregating informant reports and affirms that parent-adolescent family functioning discrepancies may provide a fuller understanding, as well as better predict adolescent problem behavior outcomes, relative to individual informant reports alone (for an overview, see De Los Reyes, 2011). For example, larger discrepancies in parent-adolescent reports of parenting behaviors have been related to higher levels of adolescent depressive symptomatology and anxiety (Ohannessian et al., 1995), greater engagement in adolescent conduct behavior problems (i.e., carrying a weapon, stealing from a store; Reynolds, MacPherson, Matusiewicz, Schreiber, \& Lejuez, 2011), and negatively associated with adolescent well-being (i.e., life purpose, confidence, positive relations with others; Stuart \& Jose, 2012). De Los Reyes, Goodman, Kliewer, and Reid-Quiñones (2010) found that, relative to parentadolescent dyads who reported smaller discrepancies, parents who reported more positive levels of parental monitoring relative to their adolescent were significantly more likely to 
have youth who reported greater levels of delinquent behaviors 2 years later (De Los Reyes et al., 2010). Noteworthy, individual parent and adolescent reports alone failed to predict adolescent delinquency. Although a few studies have examined parent-adolescent family functioning discrepancies, these studies are limited such that they did not focus on HIV risk behaviors, including substance use and sexual risk behaviors, as well as did not focus on an Hispanic sample.

\section{Family Functioning in Hispanic Families: The Role of Acculturation and Youth Gender}

The role of family functioning in Hispanic adolescent HIV risk behaviors should be examined within the context of acculturation and youth gender. Although several proxies of acculturation exist (e.g., nativity status, language), one widely utilized approach refers to the levels to which Hispanics' orientation is toward American (i.e., Americanism) and Hispanic (i.e., Hispanicism) culture (Farrelly, Cordova, Huang, Estrada, \& Prado, 2013; Martinez, 2006). From this perspective, acculturation is conceptualized as a bi-dimensional process that includes both the adoption of American culture (i.e., Americanism) and the retention of country-of-origin culture (i.e., Hispanicism; Szapocznik, Kurtines, \& Fernandez, 1980), including values, beliefs, and practices. Parent-adolescent acculturation discrepancies and the potential negative impact on family functioning have become well recognized as important antecedents of Hispanic adolescent HIV risk behaviors, including substance use and sexual risk behaviors (Prado et al. 2010; Schwartz, Mayer, et al., 2012; Unger et al., 2009). For example, some studies have shown that greater parent-adolescent acculturation discrepancies have an indirect effect on Hispanic adolescent substance use likelihood (Martinez, 2006) and substance use behaviors (Unger et al., 2009) through family functioning. Additionally, these studies demonstrate that parentadolescent acculturation discrepancies have a direct effect on family functioning (Martinez, 2006; Unger et al., 2009). These studies are limited, however, such that Martinez (2006) only examined the impact of Americanism on parent reports of family functioning and likelihood to use substances, and Unger et al.'s (2009) study included adolescents' perceptions of their parents' cultural expectations and parent reports of a single indicator of family functioning (i.e., family cohesion). This study expands on these limitations by examining the influence of parent-adolescent discrepancies in both acculturation (i.e., Americanism and Hispanicism) and latent family functioning (i.e., multiple indicators) on substance use, as well as sexual risk behavior.

Equally important in family processes is to consider the role of gender. This may be especially true in Hispanic families where numerous scholars have highlighted that childrearing practices and family roles vary by gender (Falicov, 2000; Raffaelli \& Ontai, 2004; Valenzuela, 1999). Yet, few studies have examined whether and the extent to which the influence of family functioning on HIV risk behaviors varies by youth gender. For example, a recent study indicates that low levels of parent-adolescent communication increased the risk of marijuana use for Hispanic boys, but not Hispanic girls (Lac et al., 2011). This study is limited, however, such that it did not include parent and adolescent measures of family functioning nor did it examine the role of acculturation discrepancies.

\section{Current Study and Hypotheses}

The purpose of this study was to examine whether, and the extent to which, parentadolescent discrepancies in family functioning increase the risk of Hispanic youth engaging in HIV risk behaviors, including substance use and sexual risk behaviors, as well as whether and the extent to which this influence varies by acculturation and youth gender. To the best of our knowledge, there are no published studies examining whether HIV risk 
behaviors (including unprotected sex, early sex initiation, and substance use) are related to discrepancies in family functioning. In fact, a search was conducted in PubMed, PsycINFO, and ProQuest using the keywords "discrepancies", "family functioning", "HIV", and "substance use" and no studies were identified. Given that larger parent-adolescent discrepancies in perceived family functioning have been found to increase adolescent risk for both internalizing and externalizing problems (Ohannessian et al., 1995; Reynolds et al., 2011; Stuart \& Jose, 2012), combined with previous studies indicating that lower levels of family functioning increase the risk for Hispanic adolescent HIV risk behaviors (Córdova et al., 2011; Prado et al. 2010), we extend this logic to hypothesize that greater parent-adolescent discrepancies in family functioning will increase the risk of Hispanic adolescent HIV risk behaviors. Specifically, we expect greater parent-adolescent discrepancies in family functioning (i.e., positive parenting, parental involvement, family cohesion, family communication, parental monitoring of peers, and parent-adolescent communication) will be related to higher levels of substance use (i.e., lifetime and past 90-day alcohol and drug use) and sexual risk behaviors (i.e., unprotected sex and early sex initiation). In addition, given that previous studies have suggested that acculturation discrepancies have an influence on family functioning, which in turn, increase the risk of substance use (Unger et al., 2009) and likelihood to use (Martinez, 2006), we hypothesize that greater acculturation discrepancies will indirectly increase the risk of HIV risk behaviors through family functioning. Finally, based on literature indicating gender differences in parenting practices and family roles (Lac et al., 2011), these influences are expected to vary by gender.

\section{METHOD}

This study utilized data from baseline assessments of a large randomized controlled trial conducted in collaboration with the Miami Dade County Public School system (MDCP-S). This ongoing trial is examining the relative effectiveness of Familias Unidas, a Hispanic-specific, culturally responsive intervention with demonstrated efficacy in preventing and reducing HIV risk behaviors, including substance use and sexual risk behaviors (Prado, Córdova et al., 2012; Prado, Pantin et al. 2012). This study was approved by both the University of Miami and MDCP-S Institutional Review Board.

\section{Participants}

The study sample consisted of 746 Hispanic 8th grade youth and their primary caregivers recruited from middle schools in the MDCP-S. To be eligible for this study, youth had to: (a) identify as Latino/Hispanic; (b) attend 8th grade at baseline; (c) live with an adult primary caregiver who was willing to participate; (d) be within one of the 24 cohorts of students recruited from schools in MDCP-S; and (e) plan to live in South Florida for the duration of the study.

Of the 746 Hispanic adolescents, $47.9 \%$ of the sample was female $(n=357)$ and $52.1 \%$ male $(n=389)$. The mean adolescent age was 13.9 years $(S D=0.67)$ and a slight majority of the adolescents $(55.1 \% ; n=411)$ reported being U.S. born. Foreign-born adolescents were born primarily in Cuba (40.6\%), Colombia (13.7\%), Argentina (6.0\%), and Nicaragua (5.7\%). Of foreign-born adolescents, $38.2 \%$ had been living in the United States for less than or equal to 3 years, followed by $32.2 \%$ for $3-9$ years, and $29.6 \%$ for more than 9 years. With respect to the parents, $87 \%$ of the sample was female and $13 \%$ male. The mean age of parents was 41.0 years $(S D=6.3)$ and as per the study's inclusion criteria all of the parents reported being foreign born. Participants were primarily low income families with only $32.9 \%$ of the families reporting household incomes greater than $\$ 30,000$ 
annually and $13.5 \%$ of the families reporting household incomes greater than $\$ 50,000$ annually. Parents reported a median income of $\$ 15,000$ to $\$ 20,000$.

\section{Measures}

Family functioning was assessed using adolescent and parent reports of six indicators, including positive parenting, parental involvement, family cohesion, family communication, parental monitoring of peers, and parent-adolescent communication. Positive parenting (9 items, adolescent report $\alpha=.82$, parent report $\alpha=.71$ ) and parental involvement (16 items, adolescent report $\alpha=.84$, parent report $\alpha=.76$ ) were assessed using the corresponding subscales from the Parenting Practices Scale (Gorman-Smith, Tolan, Zelli, \& Huesmann, 1996). The positive parenting subscale measures parent behaviors characterized by rewarding and acknowledging adolescent positive behaviors. Sample items for the adolescent include "When you have done something that your parents like or approve of, how often does your mother say something nice about it?", and for the parent, "When your child has done something that you like or approve of, do you say something nice about it; praise or give approval?" The response choices ranged from " $0=$ never" to " 4 = always." Parental involvement included adolescent questions such as "How often do you and your mom do things together at home?", and for the parent, "Do you and your child do things together at home?", with a response range of " $0=$ never to " $4=$ always." Family cohesion (6 items, adolescent report $\alpha=.81$, parent report $\alpha=.75$ ) and family communication (3 items, adolescent report $\alpha=.72$, parent report $\alpha=.68$ ) were assessed using the corresponding subscales from the Family Relations Scale (Tolan, Gorman-Smith, Huesmann, \& Zelli, 1997). Sample questions included "We can easily think of things to do together as a family" and "My family knows what I mean when I say something." Response choices ranged from " 1 = not true at all" to " 3 = true a lot." Parental monitoring of peers (5 items, adolescent report $\alpha=.81$, parent report $\alpha=.86$ ) was measured using the Parent Relationship with Peer Group Scale (Pantin, 1996), which asks parents to indicate the extent to which they supervise adolescents' friends, activities, and whereabouts. A fivepoint Likert-type scale, ranging from " $1=$ not at all" to " $5=$ extremely well (often)," was used to record responses. Sample adolescent items included "How well do you personally know your child's best friends?", and for parents, "How well do your parents know your best friends?" Parent-adolescent communication (20 items, adolescent report $\alpha=.90$, parent report $\alpha=.82$ ) was assessed using the Parent-Adolescent Communication Scale (Barnes \& Olson, 1985). A sample question included "I can discuss my beliefs with my mother/father without feeling restrained or embarrassed." Response choices ranged from " 1 = strongly disagree" to " 5 = strongly agree."

Acculturation was assessed using parent and adolescent reports of the Bicultural Involvement Questionnaire-Revised (BIQ-R; Birman, 1998) scale. The BIQ-R assesses the level of orientation toward American and Hispanic cultures in terms of both comfort with and enjoyment of American and Hispanic cultural practices (e.g., comfort and use of language, food, and traditions). Eleven Americanism/Hispanicism orientation items were used for parents and adolescents. Cronbach's $\alpha$ estimates for the Americanism orientation scores were .95 and .89 for parents and adolescents, respectively. Cronbach's $\alpha$ estimates for the Hispanicism scores were .85 and .91 for parents and adolescents, respectively. The parent-adolescent Americanism orientation gap score was computed by subtracting the parent's American orientation score from the adolescent's American orientation score. The parent-adolescent Hispanicism gap score was computed by subtracting the adolescent's Hispanicism score from the parents' Hispanicism score. Sample items include "I speak English at home" and "I enjoy Hispanic-oriented places". Response choices ranged from " 1 = not at all" to " 5 = very much." 
HIV risk behaviors were measured in terms of adolescent past 90-day and lifetime alcohol and drug use, unprotected sex, and early sex initiation. Adolescent alcohol and drug use was assessed using items similar to those used in the Monitoring the Future study, a national epidemiologic study to assess the prevalence of alcohol and other drug use (Johnston et al., 2012). For the purposes of this study, binary variables were created to indicate whether adolescents had ever drunk alcohol, or used an illicit drug in the past 90 days or throughout their lifetime. Early sex initiation was measured by one item that asked "Have you ever had vaginal, anal, or oral sex?" Unprotected sex was measured by one item that asked "Have you ever had vaginal or anal sex without using a condom?"

\section{Analytic Plan}

The analytic plan proceeded in two stages. In stage I, we first conducted analyses for each family functioning indicator (i.e., positive parenting, parental involvement, family cohesion, family communication, parental monitoring of peers, and parent-adolescent communication). Discrepancy scores were constructed by subtracting adolescent-reported scores of each family functioning indicator from the respective parent-reported scores. As a result, positive values indicated that the parent reported a higher score, relative to the adolescent. Conversely, negative values indicated that the adolescent reported higher scores as compared to the parent. A paired samples t test was conducted to compare parent- and adolescent-reported scores. Separate univariate regression analyses were conducted to examine whether adolescents who reported HIV risk behaviors, including alcohol and drug use and sexual risk behaviors, had higher parent-adolescent discrepancy scores for family functioning indicators.

In order to consider the multidimensional nature of family functioning, in stage II of the analytic plan, we first conducted a confirmatory factor analysis (CFA) to ascertain the feasibility of collapsing the six indicators of family functioning into a latent family functioning construct. We conducted a two-factor CFA model with adolescent-reported and parent-reported family functioning together. The fit of the hypothesized family functioning measurement model (Figure 1) was evaluated using the comparative fit index (CFI),

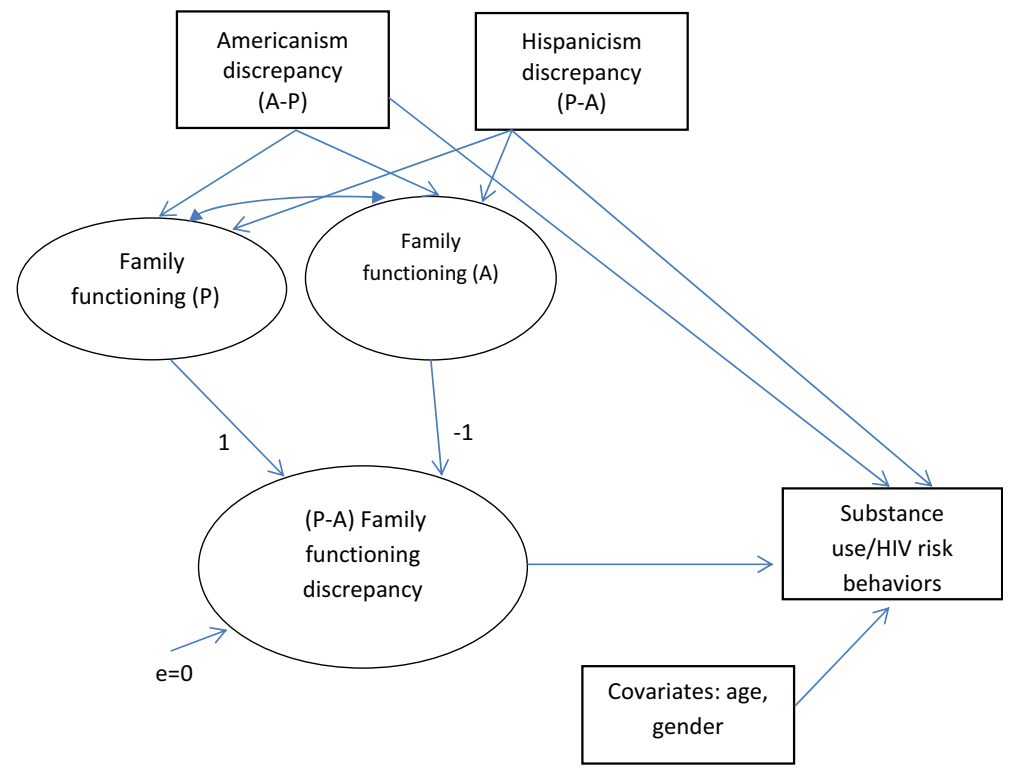

Figure 1. Hypothesized Model: The Impact of Parent-Adolescent Family Functioning Discrepancy on Hispanic Adolescent HIV Risk Behaviors 
the root mean square error of approximation (RMSEA), and the Chi-square $\left(\chi^{2}\right)$ statistic. The CFI compares the hypothesized model to a null model and values of .95 or greater indicate good model fit (Hu \& Bentler, 1999). The RMSEA estimates the deviation of the specified covariance matrix in the hypothesized model with the covariance matrix in the data and values of .06 or less indicate good model fit (Hu \& Bentler, 1999). Though the $\chi^{2}$ statistic is reported, it was not used in the interpretation as it tests the null hypothesis of perfect fit to the data, which is almost always false (Preacher, Cai, \& MacCallum, 2007).

The analytic approach for the latent parent-adolescent discrepancies was conducted as a structural equation model, which can be conceptualized as an expansion of the latent difference score method (McArdle \& Nesselroade, 1994). A latent discrepancy variable was computed that represented the latent difference of the two latent variables (i.e., parentreported family functioning and adolescent-reported family functioning) as a structural equation model (Figure 1). The latent difference was created by fixing the regression coefficient of parent-reported family functioning at 1 and fixing the adolescent-reported family functioning at -1 . The variance of the latent difference was also fixed at 0. Structural equation modeling was conducted to examine whether, and the extent to which, the latent parent-adolescent family functioning discrepancy scores significantly predicted adolescent-reported past 90-day and lifetime alcohol and drug use, early sex initiation, and unprotected sex behaviors. Important covariates such as the adolescent's age and gender were controlled for when examining these relationships.

Acculturation discrepancy scores were constructed by subtracting parent-reported scores of each Americanism from the respective adolescent-reported scores, and subtracting adolescent-reported scores of each Hispanicism from the respective parent-reported scores. Structural equation modeling was conducted to examine whether, and the extent to which, the parent-adolescent acculturation discrepancy scores were indirectly related to adolescent-reported past 90-day and lifetime alcohol and drug use, early sex initiation, and unprotected sex behaviors, through family functioning. Important covariates such as age and gender were controlled for when examining these relationships.

\section{Post Hoc Analyses by youth gender}

To determine whether the hypothesized model (see Figure 1) varied by youth gender (male as compared to female), model invariance was tested by youth gender. First, we estimated a multigroup SEM model with all parameters (i.e., path coefficients, factor loadings) freely estimated. Next, a series of multigroup SEM models were conducted first with family functioning indicator loadings constrained, acculturation discrepancies to family functioning path coefficients constrained, acculturation discrepancies to outcome path coefficients constrained, and family functioning discrepancies to outcome path coefficients constrained. Model invariance was tested by a chi-square difference test based on loglikelihood values and scaling correction factors obtained with the MLR estimator. If the difference in chi-square between the constrained and unconstrained models was nonsignificant across youth gender, a next step would be to constrain the parameters by youth gender. If the difference in chi-square between the constrained and unconstrained models was significant across youth gender, then we would freely estimate the parameters by youth gender.

Due to cluster sampling (i.e., students were clustered within schools), all of the analyses were performed using Mplus (version 7) with adjustment for complex survey design effects.

\section{RESULTS}

As can be seen in Table 1, descriptive analyses for each indicator of parent and adolescent reports of family functioning (i.e., positive parenting, parental involvement, family 
TABLE 1

Descriptive Analyses for Parent and Adolescent Reports of Family Functioning Indicators

\begin{tabular}{|c|c|c|c|c|}
\hline & Mean & $S D$ & Min & Max \\
\hline \multicolumn{5}{|c|}{ Positive parenting } \\
\hline $\mathrm{p}$ & 23.83 & 4.55 & 9.00 & 33.00 \\
\hline a & 20.16 & 6.65 & 0.00 & 33.00 \\
\hline \multicolumn{5}{|c|}{ Parental involvement } \\
\hline $\mathrm{p}$ & 43.85 & 6.42 & 17.00 & 58.00 \\
\hline a & 40.50 & 9.66 & 9.60 & 62.93 \\
\hline \multicolumn{5}{|c|}{ Family cohesion } \\
\hline $\mathrm{p}$ & 14.99 & 2.71 & 0.00 & 18.00 \\
\hline a & 12.99 & 3.39 & 2.00 & 18.00 \\
\hline \multicolumn{5}{|c|}{ Family communication } \\
\hline $\mathrm{p}$ & 7.28 & 1.72 & 0.00 & 9.00 \\
\hline a & 6.01 & 2.12 & 0.00 & 9.00 \\
\hline \multicolumn{5}{|c|}{ Peer monitoring } \\
\hline $\mathrm{p}$ & 11.71 & 4.71 & 0.00 & 20.00 \\
\hline a & 9.40 & 4.77 & 0.00 & 20.00 \\
\hline \multicolumn{5}{|c|}{ Parent-adolescent communication } \\
\hline $\mathrm{p}$ & 79.19 & 10.28 & 34.00 & 100.00 \\
\hline a & 69.89 & 14.79 & 20.00 & 100.00 \\
\hline
\end{tabular}

cohesion, family communication, parental monitoring of peers, and parent-adolescent communication) are summarized. Paired samples t tests indicate that all parent-reported family functioning indicator scores were significantly higher as compared to adolescentreported indicator scores (all $p$ values $<.001$ ).

As can be seen in Table 2, the analysis indicated that adolescents who reported illicit drug use both in their lifetime and the past 90 days had higher parent-adolescent discrepancies on all family functioning indicators, with the exception of parental peer monitoring. In addition, youth who reported having drunk alcohol both in their lifetime and the past 90 days had higher parent-adolescent discrepancies in parental involvement and parentadolescent communication. Adolescents who reported early sex initiation and unprotected sex behavior had higher parent-adolescent discrepancies in parental involvement and positive parenting.

TABLE 2

Univariate Regression Analysis of the Difference of Discrepancy Score on Family Functioning Indicators Between Adolescents Who Did and Did Not Engage in Risky Behaviors

\begin{tabular}{|c|c|c|c|c|c|c|}
\hline $\begin{array}{l}\text { Parent- } \\
\text { adolescent (p-a) }\end{array}$ & $\begin{array}{l}\text { Drug use } \\
\text { lifetime }\end{array}$ & $\begin{array}{l}\text { Drug use } \\
90 \text { days }\end{array}$ & $\begin{array}{l}\text { Alcohol } \\
\text { use } \\
\text { lifetime }\end{array}$ & $\begin{array}{l}\text { Alcohol } \\
\text { use } \\
90 \text { days }\end{array}$ & $\begin{array}{l}\text { Early sex } \\
\text { initiation }\end{array}$ & $\begin{array}{l}\text { Unprotected } \\
\text { sex }\end{array}$ \\
\hline $\begin{array}{l}\text { Positive parenting } \\
\mathrm{p}-\mathrm{a}\end{array}$ & $2.88^{*}$ & $4.57^{*}$ & $1.26^{\dagger}$ & 0.22 & $1.95^{\dagger}$ & $2.61^{*}$ \\
\hline $\begin{array}{l}\text { Parental } \\
\text { involvement p-a }\end{array}$ & $3.52 *$ & $3.33^{*}$ & $2.98^{*}$ & $3.27 *$ & $3.12^{*}$ & $3.55^{*}$ \\
\hline Family cohesion $\mathrm{p}-\mathrm{a}$ & $2.16^{*}$ & $2.47 *$ & $1.48^{*}$ & 1.43 & $1.29^{\dagger}$ & 0.46 \\
\hline $\begin{array}{l}\text { Family } \\
\text { communication } \mathrm{p}-\mathrm{a}\end{array}$ & $1.27 *$ & $1.06^{*}$ & 0.52 & $0.72^{\dagger}$ & $0.72^{\dagger}$ & 0.25 \\
\hline $\begin{array}{l}\text { Parental monitoring } \\
\text { of peers } \mathrm{p}-\mathrm{a}\end{array}$ & 0.19 & 0.79 & $0.87^{\dagger}$ & 0.47 & 0.66 & -0.65 \\
\hline $\begin{array}{l}\text { Parent-adolescent } \\
\text { communication } \mathrm{p}-\mathrm{a}\end{array}$ & $7.16^{*}$ & $7.48^{*}$ & $6.75^{*}$ & $5.59 *$ & $5.53 *$ & 1.80 \\
\hline
\end{tabular}

${ }^{*} p<.05 ;{ }^{\dagger} p<.1$. 
Next, a confirmatory factor analysis was conducted to ascertain the feasibility of collapsing six indicators of family functioning into a latent parent and adolescent-reported family functioning construct. The model provided a good fit to the data, $\chi^{2}(48)=130.2$, $p<.001 ; \chi^{2} / \mathrm{df}=2.7 ; \mathrm{CFI}=.975 ; \mathrm{RMSEA}=.048$. For adolescents, these standardized loadings ranged from .49 to .80. For parents, these loadings ranged from .49 to .78.

Finally, we tested the model in Figure 1 to estimate the relationship between the latent family functioning discrepancy and HIV risk behaviors. In Table 3, the Odds Ratio (OR) of the latent family functioning discrepancy for each outcome is summarized. Findings indicate that higher latent family functioning discrepancies were significantly related to a higher likelihood of youth engaging in lifetime and past 90-day illicit drug use, alcohol use, early sex initiation, and risky sexual behavior. Furthermore, larger parent-adolescent Hispanicism discrepancies were significantly related to lower adolescent reports of family functioning $(O R=.70, p<.001)$. Moreover, larger parent-adolescent Americanism discrepancies were significantly related to a lower risk of past 90 days alcohol $(O R=.976$, $p=.015)$ and drug use $(O R=.958, p=.017)$, and lifetime alcohol $(O R=.98, p=.016)$ and drug use $(O R=.97, p=.025)$.

\section{Post Hoc Analyses by Youth Gender}

In a post hoc analysis, we examined whether, and the extent to which, these findings varied as a function of youth gender. Multigroup SEM model by youth gender with lifetime illicit drug use outcome indicates that, when compared to a freely estimated model, constraining family functioning factor loadings across youth gender did not significantly worsen the model fit, $\Delta \chi^{2}(10)=11.2, p=.34$. In addition, constraining the path coefficients from acculturation discrepancy to family functioning, $\Delta \chi^{2}(4)=4.57, p=.33$ and lifetime drug use, $\Delta \chi^{2}(1)=0.73, p=.69$ did not significantly worsen the model fit. However, constraining path coefficients from the latent family functioning discrepancy to lifetime drug use significantly worsened the model fit, $\Delta \chi^{2}(1)=5.9, p=.015$, Wald test $\chi^{2}(1)=24.5, p<0.001$. Thus, the influence of family functioning discrepancy on lifetime drug use was stronger for females $(O R=1.109,95 \% C I=1.071-1.147)$, relative to males $(O R=1.033,95 \% C I=1.001-1.067)$. For lifetime alcohol use, constraining the path coefficients from acculturation discrepancy to lifetime alcohol use outcome did not significantly worsen the model fit, $\Delta \chi^{2}(1)=0.28, p=.87$. However, constraining the path coefficients from family functioning discrepancy to lifetime alcohol use significantly worsened the model fit, $\Delta \chi^{2}(1)=4.11, p=.043$, Wald test $\chi^{2}(1)=5.17, p=.023$. Thus, the influence of family functioning discrepancy on lifetime alcohol use outcome was stronger for females $(O R=1.084,95 \% C I=1.039-1.132)$ as compared to males $(O R=1.041,95 \% C I=1.011-$ 1.073). For all other outcomes, we did not find significant differences on path coefficients

TABLE 3

The Relationship Between Family Functioning Latent Difference and Hispanic Adolescent Substance Use and Sex Risk Behaviors

Outcomes

Drug use lifetime

Drug use 90 days

Alcohol use lifetime

Alcohol use 90 days

Early sex initiation

Unprotected sex
Family functioning latent difference OR (95\% CI)
$1.07 *(1.04,1.09)$
$1.08 *(1.04,1.12)$
$1.06 *(1.02,1.09)$
$1.05 *(1.02,1.08)$
$1.06 *(1.03,1.08)$
$1.05 *(1.001,1.09)$

${ }^{*} p<.05$. 
from family functioning discrepancy to each outcome, thus no separate results by youth gender are reported.

\section{DISCUSSION}

The purpose of this study was to examine whether, and to what extent, parent-adolescent discrepancies in family functioning (i.e., family support, family communication, parent-adolescent communication, family bonding, parental monitoring of adolescents' peers, and family cohesion) have an impact on HIV risk behaviors, including past 90-day and lifetime alcohol and drug use, unprotected sex, and early sex initiation, among Hispanic adolescents. To the best of our knowledge, this is among the first studies to examine the effects of parent-adolescent discrepancies in family functioning on HIV risk behaviors in a sample of Hispanic youth. Study findings indicate that parent-adolescent discrepancies in family functioning are associated with an increase in Hispanic youth HIV risk behaviors such that greater discrepancies are related to an increase in the likelihood of youth engaging in HIV risk behaviors, including lifetime and past 90-day alcohol and drug use, unprotected sex, and early sex initiation.

Consistent with previous studies, parent-adolescent discrepancy scores were all positive, which indicate that parents perceive more favorable views of family functioning, relative to youth (Ohannessian et al., 1995; Schwartz et al., 2008; Steinberg, 1990; Stuart \& Jose, 2012). Irrespective of parent reports, study findings suggest that adolescent reports of family functioning still predict HIV risk behaviors, including lifetime and past 90-day alcohol and illicit drug use, unprotected sex, and early sex initiation. Therefore, it appears that if the research question is concerned with youth outcomes, perhaps the adolescent's perceptions, even with biases, may count more than parent's perceptions (Tein et al., 1994). Yet, as study findings indicate, parent-adolescent family functioning discrepancies may provide a fuller understanding and systemic perspective of the family system - a methodological challenge that family researchers have worked to address since its inception (Russel, 1979). Future studies should examine if these findings hold true in longitudinal designs.

With respect to substance use behaviors, findings indicate that parent-adolescent discrepancies in family functioning are associated with an increased likelihood of youth reporting both lifetime and past 90-day alcohol and illicit drug use. These findings are consistent with previous studies which show that parent-adolescent discrepancies in family functioning are associated with adolescent problem behaviors (De Los Reyes et al., 2010; Reynolds et al., 2011), and expand on this line of research by focusing on substance use outcomes in a sample of Hispanic youth. These findings are particularly relevant as (a) alcohol remains the most widely used substance by youth today; (b) adolescent reports of having used any illicit drug have increased over the past 3 years; and (c) Hispanic youth constitute a population that reports the highest rates of lifetime and current alcohol and illicit drug use (with the exception of amphetamines; Johnston et al., 2012).

Previous studies indicate that intrapersonal risk, irrespective of ecodevelopmental risk (i.e., family functioning), is a better predictor of sexual risk behaviors in Hispanic youth (Prado et al., 2009). Although this study did not focus on intrapersonal factors, contrary to previous research, our findings suggest that parent-adolescent discrepancies in family functioning, an ecodevelopmental risk, are associated with sexual risk behaviors. Specifically, parent-adolescent discrepancies in family functioning are related to unprotected sex and early sex initiation. Thus, it may be that for ecodevelopmental family risk, what is important is the discrepancy and not the absolute value of family functioning.

Not surprisingly, findings suggest that larger parent-adolescent Hispanicism discrepancies were significantly related to a decrease in adolescent reports of family 
functioning. This finding confirms previous studies which indicate that acculturation discrepancies negatively affect family functioning among Hispanics (Gil, Wagner, \& Vega, 2000; Santisteban, Coatsworth, Briones, Kurtines, \& Szapocznik, 2012; Schwartz et al., 2013). In addition, our findings indicate that larger parent-adolescent Americanism discrepancies were significantly related to a decreased risk of past 90 days and lifetime alcohol and drug use. These findings are similar to previous research which suggests that Americanism discrepancies are associated with a decreased likelihood of substance use behaviors (Schwartz, Unger et al., 2012). According to Schwartz et al. (2013), adapting American cultural norms and practices does not seem to negatively impact family functioning and increase the risk for HIV risk behaviors as long as Hispanic youth retain Hispanic cultural norms and practices. From a developmental perspective, future research should examine trajectories of Hispanic parent-adolescent acculturation and family functioning discrepancies to develop a fuller understanding of these processes over time.

Researchers have found that lower levels of parental communication are associated with an increased risk for marijuana use for Hispanic males, but not Hispanic females (Lac et al., 2011). Contrary to these findings, our research indicates that the effects of family functioning discrepancies on lifetime alcohol and drug use are stronger among Hispanic females, relative to males. One potential explanation of this finding refers to the fact that Hispanic females and males develop in different family contexts shaped by different parenting practices, gender roles, and expectations. For example, relative to females, Hispanic males are often expected to develop skills related to autonomy and self-sufficiency outside of the family context (Falicov, 2013; Raffaelli \& Ontai, 2004; Valenzuela, 1999). Thus, it may very well be that, during this developmental period, other contexts (e.g., peer/school) could be more influential in Hispanic males, relative to females. Future research should examine the influence of additional ecodevelopmental contexts on HIV risk behaviors over time.

From an intervention perspective, there still remains the need to develop optimally efficacious and culturally relevant family-based preventive interventions (Parra-Cardona et al., 2012; Parra-Cardona, Córdova, Holtrop, Villarruel, \& Wieling, 2008). This study contributes to this need because discrepancies in informants' reports can be used to identify meaningful preventive intervention outcomes (De Los Reyes, 2011). Indeed, parentadolescent discrepancies are ubiquitous in all families in the United States. It should not be surprising then that our study suggests that parents and adolescents differ with respect to how they perceive family functioning. These discrepancies, however, may be more pronounced in Hispanic families where they also face the challenges of acculturation discrepancies. Future research on family-based interventions targeting multiple dimensions of family functioning can examine whether a tailored version aimed at narrowing parent-adolescent discrepancies is more efficacious in preventing/reducing HIV risk behaviors, relative to a general family functioning version. Although a substantial amount of research has demonstrated that family functioning is a pathway through which interventions can prevent and reduce HIV risk behaviors among Hispanic youth (Córdova et al., 2012; Prado, Córdova et al., 2012; Sale et al., 2005; Sandler et al., 2011), there are no studies that examine whether, and to what extent, parent-adolescent discrepancies in family functioning mediate the impact of family-based preventive interventions on HIV risk behaviors. Indeed, randomized controlled trial outcomes vary as a function of the usage of informants, methodological approaches, and statistical analysis (Achenbach, 2011; Huang et al., in press). Given that parent-adolescent discrepancies in family functioning constitute a malleable ecodevelopmental risk factor (Prado, Córdova et al., 2012), a better understanding of this phenomenon may have great utility in working toward optimally efficacious and effective family-based prevention programs. 
This study has important clinical implications. Family therapists who seek to reduce Hispanic adolescent HIV risk behaviors could focus their practice efforts on minimizing parent-adolescent discrepancies with respect to how parents and adolescents differentially perceive the family environment. For example, parents who report significantly higher on family functioning, relative to their child, could benefit from family therapists interviewing the family to probe the basis for these discrepancies (Achenbach, 2011). Study findings, for example, demonstrate that parents reported higher parent-adolescent communication, as compared to the youth. Family therapists can, therefore, show the parent and adolescent their respective scores on parent-adolescent communication and explore the ways in which parent-adolescent communication can be improved (Achenbach, 2011). Studies show superior treatment gains when both the parent and adolescent report a problem (De Los Reyes, 2011), and this approach may be helpful to demonstrate to the family potential areas for improvement. This, in and of itself, can be seen as an intervention because this approach has the potential to build therapeutic alliance, improve communication around family functioning discrepancies, and introduce other family therapy techniques such as sculpting (Papp, Scheinkman, \& Malpas, 2013; Papp, Silverstein, \& Carter, 1973; Simon, 1972) to complement these discussions and further highlight each client's perspective of how well the family functions.

In this study, the use of parent-adolescent discrepancies on both family functioning and a bi-dimensional model of acculturation constitute parsimonious alternatives (McArdle, 2001) and are important methodological advances in the family process literature. Yet, some scholars may still pose the question: Are discrepancies meaningful? In spite of the fact that many family researchers have access to parent-adolescent reports, few have attempted to empirically answer questions to better understand the ways in which discrepancies in parent-adolescent reports play an important role in the etiology of adolescent problem behaviors. In fact, family researchers traditionally have treated parentadolescent discrepancies as a methodological artifact, nuance, or indirect construct (Achenbach, 2011; De Los Reyes, 2011). More recently, however, this important construct has moved from being ignored, to being accounted for as an indirect construct, to being examined as a direct construct in empirical research (De Los Reyes, 2011). Latent discrepancy scores provide advanced formal ways of empirically testing questions that family researchers routinely ask, but rarely answer, as well as novel approaches to answering old questions (McArdle, 2001). Similar to previous research examining informant discrepancies (Achenbach, McConaughy, \& Howell, 1987; Hartley, Zakriski, \& Wright, 2011), our study provides provocative initial data which suggest that, yes, indeed, parent-adolescent discrepancies are meaningful and reveal important information about Hispanic adolescent HIV risk behaviors. These findings are an important step in working toward a fuller understanding of etiological factors, including parent-adolescent discrepancies in family functioning, to help curb the tide of HIV inequities experienced by this population. With the advancement of methodological and statistical analysis, perhaps now more than ever, researchers are better situated to answer empirical questions related to parent-adolescent discrepancies in longitudinal multivariate designs. We should only accept the argument that parent-adolescent discrepancies do not provide family researchers and clinicians with meaningful information to ameliorate HIV risk behaviors in this population when such data exist to support this position (De Los Reyes, 2011).

Findings should be interpreted in light of several study limitations. First, the study utilized a cross-sectional design. Therefore, causality with respect to the impact of parentadolescent discrepancies on adolescent HIV risk behaviors cannot be inferred. Future studies should include a longitudinal research design to work toward a better understanding of the effects of parent-adolescent family functioning discrepancies on Hispanic adolescent HIV risk behaviors. Second, the study sample is not representative of the U.S. 
Hispanic adolescent population and thus findings are not generalizable to all U.S. Hispanic youth. Third, self-report measures were employed in this study. Thus, it is possible that participants may over- or underreport substance use and sexual risk behaviors.

In summary, this is among the first studies to examine the impact of parent-adolescent family functioning discrepancies on Hispanic adolescent HIV risk behaviors. Study findings indicate that parent-adolescent family functioning discrepancies may increase the risk of lifetime and past 90-day alcohol and illicit drug use and early sex initiation. Findings are suggestive that family-based preventive interventions aimed at narrowing parent-adolescent family functioning discrepancies may aid in preventing and reducing Hispanic adolescent HIV risk behaviors. The ultimate goal of these efforts should be to eliminate HIV health disparities and work toward health equity in this population.

\section{REFERENCES}

Achenbach, T. M. (2006). As others see us: Clinical and research implications of cross-informant correlations for psychopathology. Current Directions in Psychological Science, 15, 94-98.

Achenbach, T. M. (2011). Commentary: Definitely more than measurement error: But how should we understand and deal with informant discrepancies? Journal of Clinical Child \& Adolescent Psychology, 40(1), 80-86.

Achenbach, T. M., McConaughy, S. H., \& Howell, C. T. (1987). Child/adolescent behavioral and emotional problems: Implications of cross-informant correlations for situational specificity. Psychological Bulletin, 101(2), 213.

Barnes, H. L., \& Olson, D. H. (1985). Parent-adolescent communication and the circumplex model. Child Development, $56,438-447$.

Birman, D. (1998). Biculturalism and perceived competence of Latino immigrant adolescents. American Journal of Community Psychology, 26(3), 335-354.

Centers for Disease Control \& Prevention (2012a). Youth risk behavior surveillance-United States, 2011. MMWR Surveillance Summaries, 61(SS-04), 1-162. Retrieved from http://www.cdc.gov/mmwr/pdf/ss/ss6104.pdf (accessed on May 3, 2013)

Centers for Disease Control \& Prevention. (2012b). HIV among Latinos. Retrieved from http://www.cdc.gov/hiv/ pdf/risk_latino.pdf (accessed on May 3, 2013).

Córdova, D., Huang, S., Arzon, M., Freitas, D., Malcolm, S., \& Prado, G. (2011). The role of attitudes, family, peer and school on alcohol use, rule breaking and aggressive behavior in Hispanic delinquent adolescents. Open Family Studies Journal, 4, 38-45.

Córdova, D., Huang, S., Pantin, H., \& Prado, G. (2012). Do the effects of a family intervention on alcohol and drug use vary by nativity status? Psychology of Addictive Behaviors, 26, 655-660.

De Los Reyes, A. (2011). Special section: More than measurement error: Discovering meaning behind informant discrepancies in clinical assessments of children and adolescents. Journal of Clinical Child \& Adolescent Psychology, 40, 1-9.

De Los Reyes, A., Goodman, K. L., Kliewer, W., \& Reid-Quiñones, K. R. (2010). The longitudinal consistency of mother-child reporting discrepancies of parental monitoring and their ability to predict child delinquent behaviors two years later. Journal of Youth and Adolescence, 39, 1417-1430.

Falicov, C. J. (2013). Latino families in therapy. New York, NY: Guilford Press.

Farrelly, C., Córdova, D., Huang, S., Estrada, Y., \& Prado, G. (2013). The role of acculturation and family functioning in predicting HIV risk behaviors among Hispanic delinquent youth. Journal of Immigrant and Minority Health, 13, 1-8.

Gil, A. G., Wagner, E. F., \& Vega, W. A. (2000). Acculturation, familism, and alcohol use among Latino adolescent males: Longitudinal relations. Journal of Community Psychology, 28(4), 443-458.

Gorman-Smith, D., Tolan, P. H., Zelli, A., \& Huesmann, L. R. (1996). The relation of family functioning to violence among inner-city minority youths. Journal of Family Psychology, 10, 115-129.

Hartley, A. G., Zakriski, A. L., \& Wright, J. C. (2011). Probing the depths of informant discrepancies: Contextual influences on divergence and convergence. Journal of Clinical Child \& Adolescent Psychology, 40(1), 54-66.

Hu, L., \& Bentler, P.M. (1999). Cutoff criteria for fit indexes in covariance structure analysis: Conventional criteria versus new alternatives. Structural Equation Modeling, 6, 1-55.

Huang, S., Córdova, D., Estrada, Y., Brincks, A., Asfour, L., \& Prado, G. (in press). An application of the complier average causal effect analysis to examine the effects of a family intervention in reducing illicit drug use among high-risk Hispanic adolescents. Family Process.

Johnston, L. D., O’Malley, P. M., Bachman, J. G., \& Schulenberg, J. E. (2012). Monitoring the Future national survey results on drug use, 1975-2011: Volume I, Secondary school students. Ann Arbor, MI: Institute for Social Research, The University of Michigan. 
Lac, A., Unger, J. B., Basáñez, T., Ritt-Olson, A., Soto, D. W., \& Baezconde-Garbanati, L. (2011). Marijuana use among Latino adolescents: Gender differences in protective familial factors. Substance Use \& Misuse, 46(5), 644-655.

Martinez, C. R. (2006). Effects of differential family acculturation on Latino adolescent substance use. Family Relations, 55(3), 306-317.

Martinez, C. R., \& Eddy, J. M. (2005). Effects of culturally adapted parent management training on Latino youth behavioral health outcomes. Journal of Consulting and Clinical Psychology, 73, 841-851.

McArdle, J. J. (2001). A latent difference score approach to longitudinal dynamic structural analyses. In R. Cudeck, S. du Toit \& D. Sorbom (Eds.), Structural equation modeling: Present and future (pp. 342-380). Lincolnwood, IL: Scientific Software International.

McArdle, J. J., \& Nesselroad, J. R. (1994). Using multivariate data to structure developmental change. In S. Cohen \& H. Resse (Eds.), Life-span developmental psychology: Methodological Innovations (pp. 223-268). Hillsdale, NJ: Lawrence Erlbaum.

Mouttapa, M., Weiss, J. W., \& Hermann, M. (2009). Is image everything? The role of self-image in the relationship between family functioning and substance use among Hispanic adolescents. Substance Use \& Misuse, 44, $702-721$.

Ohannessian, C. M., Lerner, R. M., Lerner, J. V., \& von Eye, A. (1995). Discrepancies in adolescents' and parents' perceptions of family functioning and adolescent emotional adjustment. Journal of Early Adolescence, 15, 490-516.

Pantin, H. (1996). Ecodevelopmental measures of support and conflict for Hispanic youth and families. Miami, FL: University of Miami School of Medicine.

Papp, P., Scheinkman, M., \& Malpas, J. (2013). Breaking the mold: Sculpting impasses in couples' therapy. Family Process, 51, 33-45. doi:10.1111/famp.12022.

Papp, P., Silverstein, O., \& Carter, E. (1973). Family sculpting in preventive work with "well families". Family Process, 12(2), 197-212.

Parra-Cardona, J. R., Córdova, D., Holtrop, K., Villarruel, F. A., \& Wieling, E. (2008). Shared ancestry, evolving stories: Similar and contrasting life experiences described by foreign born and US born Latino parents. Family Process, 47(2), 157-172.

Parra-Cardona, J. R., Domenech-Rodriguez, M., Forgatch, M., Sullivan, C., Bybee, D., Holtrop, K. et al. (2012). Culturally adapting an evidence-based parenting intervention for Latino immigrants: The need to integrate fidelity and cultural relevance. Family Process, 51(1), 56-72.

Prado, G., Córdova, D., Huang, S., Estrada, Y., Bacio, G. A., Leon Jimenez, G. et al. (2012). The efficacy of Familias Unidas on drug and alcohol use for Hispanic delinquent youth: Main effects and effects by environmental context. Drug and Alcohol Dependence, 125, S18-S25.

Prado, G., Huang, S., Maldonado-Molina, M., Bandiera, F., Schwartz, S. J., de la Vega, P. et al. (2010). An empirical test of ecodevelopmental theory in predicting HIV risk behaviors among Hispanic youth. Health Education \& Behavior, 37, 97-114.

Prado, G., Pantin, H., Briones, E., Schwartz, S., Feaster, D., Huang, S. et al. (2007). A randomized controlled trial of a family-centered intervention in preventing substance use and HIV risk behaviors in Hispanic adolescents. Journal of Consulting and Clinical Psychology, 75, 914-926.

Prado, G., Pantin, H., Huang, S., Córdova, D., Tapia, M., Velazquez, M. R. et al. (2012). Effects of a family intervention in reducing HIV risk behaviors among high-risk Hispanic adolescents: A randomized controlled trial. Archives of Pediatrics and Adolescent Medicine, 166, 127-133.

Prado, G. J., Schwartz, S. J., Maldonado-Molina, M., Huang, S., Pantin, H. M., Lopez, B. et al. (2009). Ecodevelopmental x intrapersonal risk: Substance use and sexual behavior in Hispanic adolescents. Health Education \& Behavior, 36, 45-61.

Preacher, K. J., Cai, L., \& MacCallum, R. C. (2007). Alternatives to traditional model comparison strategies for covariance structure models. In T. D. Little, J. A. Bovaird, \& N. A. Card (Eds.), Modeling contextual effects in longitudinal studies (pp. 33-62). Mahwah, NJ: Lawrence Erlbaum Associates.

Raffaelli, M., \& Ontai, L. L. (2004). Gender socialization in Latino/a families: Results from two retrospective studies. Sex Roles, 50(5-6), 287-299.

Reynolds, E. K., MacPherson, L., Matusiewicz, A. K., Schreiber, W. M., \& Lejuez, C. W. (2011). Discrepancy between mother and child reports of parental knowledge and the relation to risk behavior engagement. Journal of Clinical Child and Adolescent Psychology, 40, 67-79.

Russel, C. S. (1979). Circumplex model of marital and family systems: III. Empirical evaluation with families. Family Process, 18, 29-45.

Sale, E., Sambrano, S., Springer, J. F., Peña, C., Pan, W., \& Kasim, R. (2005). Family protection and prevention of alcohol use among Hispanic youth at high risk. American Journal of Community Psychology, 36, $195-205$.

Sandler, I. N., Schoenfelder, E. N., Wolchik, S. A., \& MacKinnon, D. P. (2011). Long-term impact of prevention programs to promote effective parenting: Lasting effects but uncertain processes. Annual Review of Psychology, 62, 299-329. 
Santisteban, D. A., Coatsworth, J. D., Briones, E., Kurtines, W., \& Szapocznik, J. (2012). Beyond acculturation: An investigation of the relationship of familism and parenting to behavior problems in Hispanic youth. Family Process, 51(4), 470-482.

Santisteban, D. A., Coatsworth, J. D., Perez-Vidal, A., Kurtines, W. M., Schwartz, S. J., LaPerriere, A. et al. (2003). Efficacy of brief strategic family therapy in modifying Hispanic adolescent behavior problems and substance use. Journal of Family Psychology, 17, 121-133.

Santisteban, D. A., \& Mena, M. P. (2009). Culturally informed and flexible family-based treatment for adolescents: A tailored and integrative treatment for Hispanic youth. Family Process, 48, 253-288.

Schwartz, S. J., Des Rosiers, S., Huang, S., Zamboanga, B. L., Unger, J. B., Knight, G. P. et al. (2013). Developmental trajectories of acculturation in Hispanic adolescents: Associations with family functioning and adolescent risk behavior. Child Development, 84, 1355-72.

Schwartz, S. J., Mason, C. A., Pantin, H., \& Szapocznik, J. (2008). Effects of family functioning and identity confusion on substance use and sexual behavior in Hispanic immigrant early adolescents. Identity, 8, $107-124$.

Schwartz, B., Mayer, B., Trommsdorff, G., Ben-Arieh, A., Friedlmeier, M., Lubiewska, K. et al. (2012). Does the importance of parent and peer relationships for adolescents' life satisfaction vary across cultures? The Journal of Early Adolescence, 32(1), 55-80.

Schwartz, S. J., Unger, J. B., Rosiers, S. E. D., Huang, S., Baezconde-Garbanati, L., Lorenzo-Blanco, E. I. et al. (2012). Substance use and sexual behavior among recent Hispanic immigrant adolescents: Effects of parentadolescent differential acculturation and communication. Drug and Alcohol Dependence, 125, S26-S34.

Simon, R. M. (1972). Sculpting the family. Family Process, 11(1), 49-57.

Steiger, J. H. (1990). Structural model evaluation and modification: An interval estimation approach. Multivariate Behavioral Research, 25, 173-180.

Steinberg, L. D. (1990). Autonomy, conflict and harmony in the family relationship. In S. S. Feldman \& G. R. Elliott (Eds.), At the threshold: The developing adolescent (pp. 255-276). Cambridge, MA: Harvard University Press.

Stuart, J., \& Jose, P. E. (2012). The influence of discrepancies between adolescent and parent ratings of family dynamics on the well-being of adolescents. Journal of Family Psychology, 26, 858-868.

Sullivan, S., Schwartz, S. J., Prado, G., Huang, S., Pantin, H., \& Szapocznik, J. (2007). A bidimensional model of acculturation for examining differences in family functioning and behavior problems in Hispanic immigrant adolescents. The Journal of Early Adolescence, 27, 405-430.

Szapocznik, J., Kurtines, W. M., \& Fernandez, T. (1980). Bicultural involvement and adjustment in HispanicAmerican youths. International Journal of Intercultural Relations, 4(3), 353-365.

Szapocznik, J., Prado, G., Burlew, A. K., Williams, R. A., \& Santisteban, D. A. (2007). Drug abuse in African American and Hispanic adolescents: Culture, development, and behavior. Annual Review of Clinical Psychology, 3, 77-105.

Tein, J. Y., Roosa, M. W., \& Michaels, M. (1994). Agreement between parent and child reports on parental behaviors. Journal of Marriage and the Family, 56, 341-355.

Tolan, P. H., Gorman-Smith, D., Huesmann, L. R., \& Zelli, A. (1997). Assessment of family relationship characteristics: A measure to explain risk for antisocial behavior and depression among urban youth. Psychological Assessment, 9, 212-223.

Unger, J. B., Ritt-Olson, A., Wagner, K. D., Soto, D. W., \& Baezconde-Garbanati, L. (2009). Parent-child acculturation patterns and substance use among Hispanic adolescents: A longitudinal analysis. Journal of Primary Prevention, 30, 293-313.

Valenzuela, A. (1999). Gender roles and settlement activities among children and their immigrant families. American Behavioral Scientist, 42(4), 720-742. 